\title{
Analysis of Manhattan mobility model without RSUs
}

\author{
Dr. B. Ramakrishnan Ph.D \\ Department of Computer Science and Research centerS.T.Hindu collegeNagercoil-629002
}

\begin{abstract}
The vehicular communication is an important issue to the researchers who are engaged in preventing traffic accidents and traffic jams. The earlier vehicular models had discussed only communication among vehicles through the Road Side Units (RSU). Most of the researchers used IEEE 802.11 for vehicular communication in which the vehicles are moving inside the city [1]. But in this paper the author uses the latest VANET technology 802.11p in the Manhattan mobility model in which the nodes are moving inside the city [2]. Without using the RSUs, each vehicle in the Manhattan mobility network is treated as a router to communicate with the neighboring vehicles. The standard VANET routing protocols are applied to the Manhattan mobility model and their characteristics are compared with the use of NS 2.34 version simulator and their results are presented in this work [3].
\end{abstract}

Keywords: VANET, MANET, RSUs, AODV, DSDV, DSR, 802.11, 802.11p

\section{Introduction}

Today communication networks are many and varied. More and more research is going on in this area. Every day there comes a new technology, with advanced features in its structure and operations. The devices look lean and thin, but their computing performance is immense. The fast growth of these devices and the popular use of them in communication necessitate the deployment of wireless network communication [4].

When the source and destination nodes may not be within the data communication range then the path between them are multihop [5]. All the nodes in the network act as a router to forward the packets to the other nodes to provide the data communication throughout the network. Topology changes occur due to the mobility of the nodes, the adjustment of transmission and reception parameters and the sleep cycles of nodes to save energy. Therefore the network topology may change frequently at unpredictable times. The most notable characteristic is the dynamic topology. These characteristics create large challenges for protocols on any layers of the networks.

\subsection{Introduction}

\section{Literature Review}

Vehicular Ad hoc Network (VANET) is a special type of Intelligent Transport System (ITS), where the mobile nodes are motorcars, two wheelers, trucks, buses etc., that move on well organized and predefined roads in both direction at very high speed [6][7]. Following traffic rules, the vehicles provide communication with each other directly (Inter Vehicle Communication - IVC) or indirectly through the Road Side Unit (RSU) [8]. Most of the Mobile Adhoc network (MANET) features are applicable in the VANET environment [9]. When a survey is made on the subject it is found that the protocols and mobility models are limited. So various attempt have been made to create a new mobility model and routing protocols for VANET [10][11].

\subsection{Manhattan mobility model}

There are various mobility models in Vehicular Ad hoc networks (VANET) [12]. These mobility models guide the drivers of the vehicle in taking the right path and they also provide safety and comfort. One such mobility model is the Manhattan Mobility model.

The Manhattan mobility model is usually used to emulate the movement pattern of mobile nodes on streets defined by maps. It can be useful in modeling movement in an urban area. This model also uses its own map. The map is composed of a number of horizontal and vertical streets. Each street has two lanes for each direction (north and south direction for vertical streets, east and west for horizontal streets) [13]. The mobile node is allowed to move along the grid of horizontal and vertical streets on the map. At an intersection of a horizontal and a vertical street, the mobile node can turn left, right or go straight. This choice is probabilistic: the probability of moving on the same street is 0.5 , the probability of turning left is 0.25 and the probability of turning right is 0.25 .[] The velocity of a mobile node at a time slot is dependent on its velocity at the previous time slot. In addition, a node's velocity is restricted by the velocity of the node preceding it on the same lane of the street. It also imposes geographic restrictions on node mobility. The Manhattan map used in the simulations is shown in the following Figure 1. 


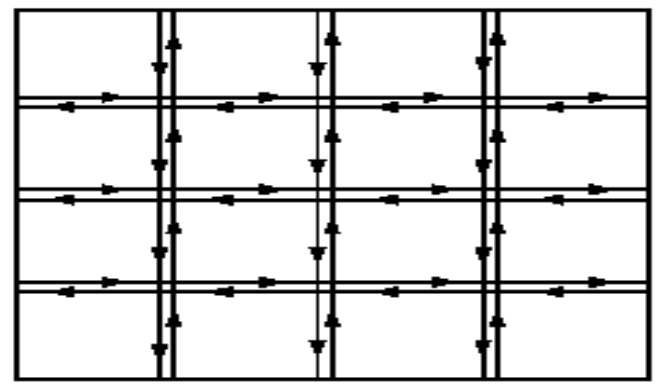

Figure 1 Map used in Manhattan Model

\subsection{Usage and description of Mobility generators}

The mobility generator tools are used to generate a set of mobility scenarios used to evaluate the protocol performance in Mobile Ad hoc network. The tools include the Freeway model and Manhattan model. The trace files generated by these tools are compatible with the format required by NS- 2 . The researcher could directly input the trace file generated by this generator in to the NS-2 simulator and run the simulation. After modifications of appropriate parameters, this set of mobility generators is able to create various mobility scenarios with different mobility characteristics.

\section{System Model}

\subsection{Manhattan mobility model with Road side unit (RSUs)}

Most of the existing Manhattan mobility models are assumed as a collection of nodes (vehicles), links and fixed units called Roadside Units (RSU). Communication at any point is done through a VANET node to another VANET node through a Fixed RSUs only [14].

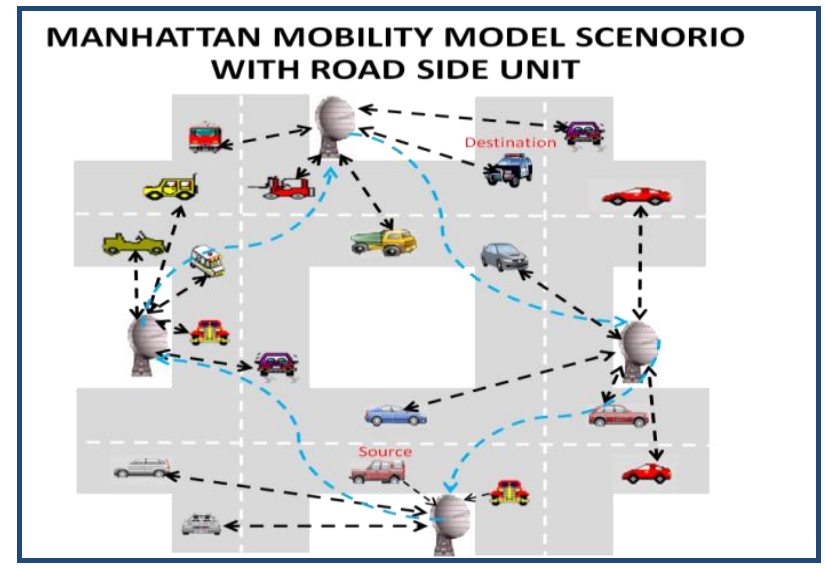

Figure 2: $\quad$ Manhattan mobility model with RSUs

When a vehicle enters a city area, it directly sends the request to the road side units and gets the responses easily. In the earlier vehicular network model all the nearby vehicles are connected to this road side units and all the road side units are in turn connected together to form mobile ad-hoc network which is shown in Figure 2. This scenario is valid only for the vehicles moving inside the city where the vehicles move slowly and more numbers of fixed base stations are available.

\subsection{Manhattan mobility model without Road side unit (RSUs)}

For effective vehicular communication each vehicle acts as a router to exchange information. Each vehicle is equipped with a global positioning system (GPS). Broadcasting and routing algorithms use the information provided by the GPS and make effective communication [15]. For the road safety, new applications are proposed for vehicular networks, i.e. car to car communication, travel and tourism information distribution and game applications. These applications need reliable communication equipment with high data rates and also a stable connectivity between the transmitter and the receiver under high reliability condition. The vehicular communication with out RSUs is shown in the Figure 3. 


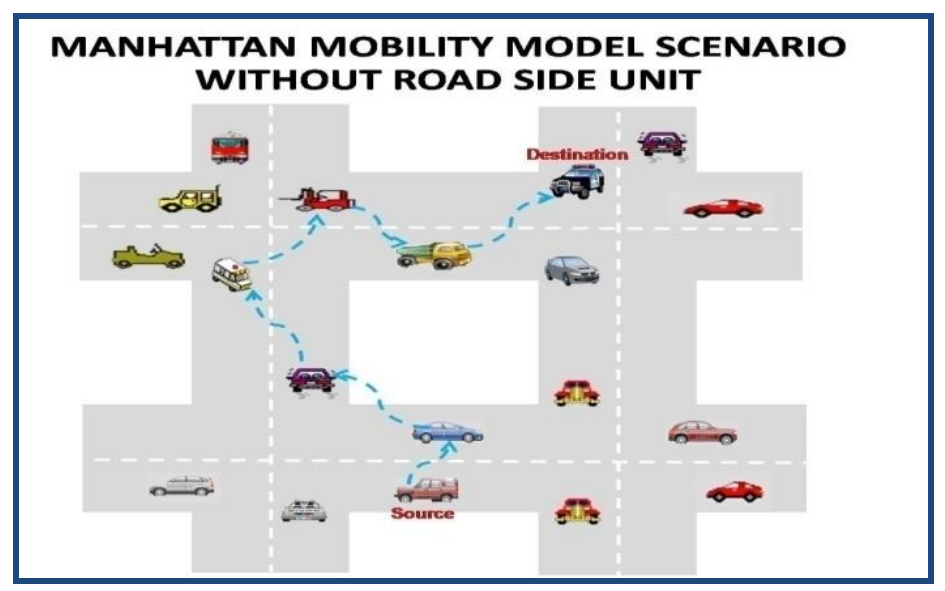

Figure 3: Manhattan mobility model without RSUs

\subsection{Road side unit in city area}

Vehicles play a more important role in transportation. However, too many vehicles may cause heavy traffic congestion in cities; more people were killed in traffic accidents. Intelligent transportation system (ITS) offers a promising solution for both traffic congestion and road safety in vehicular ad hoc networks (VANETs). Considerable research efforts have been made in VANET security issues in the past years, most of which focus on the authentication, securing positioning and route of On-Board-Units(OBUs), meanwhile a few attempts on the infrastructures communication in the VANETs are made in building a scalable system [16]. Most studies hold the assumptions that Road-Side-Unit (RSU) infrastructures will not be deployed in the near future, thus we have to taking advantage of the OBUs themselves with predefined certification to accomplish a secure VANET with little or no help of external road infrastructures.

Besides, some studies propose authentication approach that an OBU needs to authenticate itself to the RSU every time it enters the range of a RSU. Given the fact that the distance between two RSUs is about $500 \mathrm{~m}$, the vehicle's velocity is about $60 \mathrm{~km} / \mathrm{h}$ in city, so the vehicle has to perform the authentication routine every 30 seconds. Road side infrastructures occupy critically important position in the VANET system because only the road side infrastructures can build a skeleton of the whole distributed system, a most of the transaction based services are provided by roadside units. This paper presents a new approach for data communication inside the city limit where there are no road side units.

\section{Algorithm For Data Communication Without Rsus}

In wireless networks, users share a common medium, thereby creating a need for a protocol that provides efficient access. The most commonly used MAC protocols in wireless networks are Frequency Division Multiple Access (FDMA), Time Division Multiple Access (TDMA) and Carrier Sense Multiple Access with Collision Avoidance (CSMA/CA). The CSMA/CA protocol which is included in the IEEE 802.11 standard, is the most widely used MAC protocol in wireless LANs and MANETs. In this paper, 802.11 is also used in network simulation. In CSMA/CA, when a node wants to transmit a packet, it first listens to the medium. If the channel is idle then it transmits the packet to the destination node otherwise it waits for a random period of time. The Ready-to-Send (RTS) and Clear-to-Send (CTS) messages are used to reduce the collision problem, which occurs if two nodes try to access the medium simultaneously. When the channel is idle, the transmitting node sends a RTS packet containing information about the length of the message that is ready for transmission. If the receiving node hears the RTS, it will send a CTS packet, allowing the transmitter to send its packet. Finally, upon successful reception of the packet, the receiving node sends an ACK [17].

The VANET communication without RSUs is established by the routing table formation algorithm and the route discovery algorithm is shown in Algorithm 1 and Algorithm 2. In this model, the node communicates to the destination using multi-hop communication. 


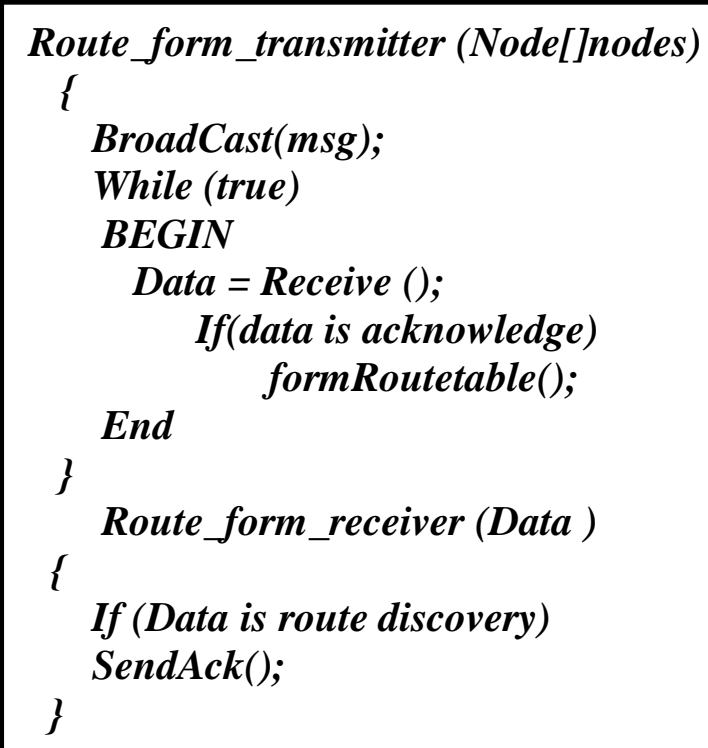

Algorithm 1: Routing table formation

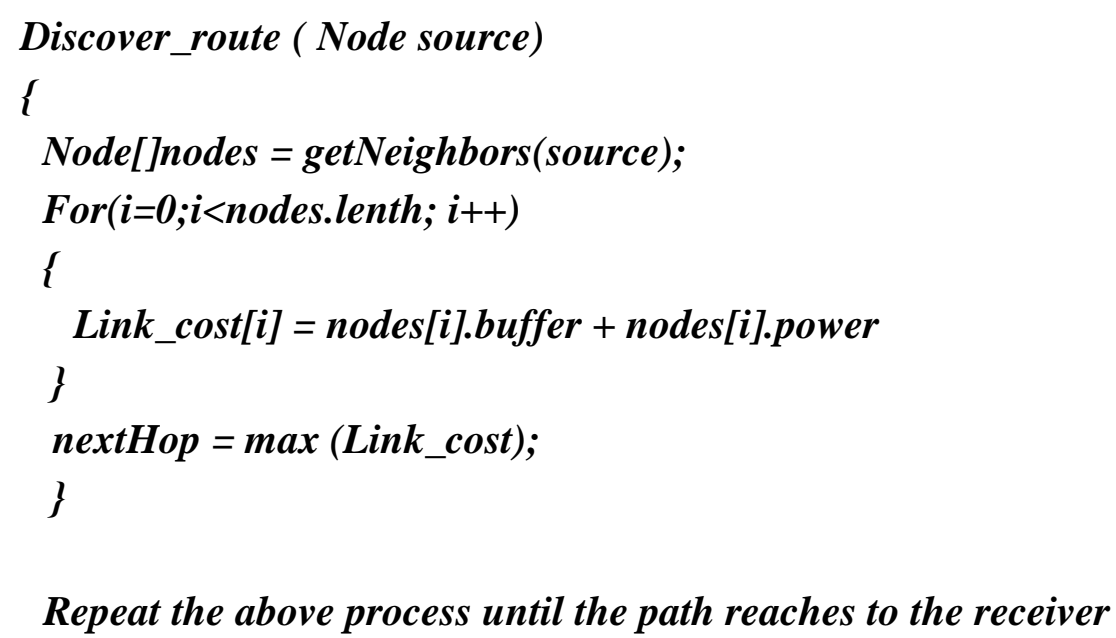

Algorithm 2: Routing Discovery

\section{Vanet Simulation}

5.1 Parameters Used In Manhattan Simulation

The parameters used in NS2.34 VANET simulation is shown in Table1.

\begin{tabular}{|l|l|}
\hline Network Area & $1500 \mathrm{~m} * 1500 \mathrm{~m}$ \\
\hline Channel Type & Wireless \\
\hline Propagation Model & Two Way Ground \\
\hline Radio Range & $200 \mathrm{~m}$ \\
\hline Radio Delay & $10 \mathrm{~ms}$ \\
\hline Traffic Type & CBR \\
\hline Visualization Tools & NAM, Tracing \\
\hline MAC Layer & IEEE 802.11, 802.11p \\
\hline Protocol & DSDV, DSR, AODV \\
\hline Mobility & MANHATTAN \\
\hline Number of Nodes & $25,50,75,100,150$ \\
\hline Speeds & $5 \mathrm{~m} / \mathrm{sec}$ \\
\hline
\end{tabular}

Table 1: Critical parameters used in NS 2.34 


\subsection{VANET IEEE standard 802.11}

In the MANET, the nodes are moving at random and their speed is normal but in the VANET, nodes are high speed moving vehicles. Due to the high speed of the vehicle nodes, their network topology changes quickly. Hence the technology 802.11 used in MANET yields less performance in VANET [18]. So the IEEE working group has developed a new PHY/MAC amendment of the 802.11 standard which is specially designed for vehicular communication. This wireless access in vehicular environment (WAVE) is known as IEEE 802.11p which is more suitable for high speed vehicle data communication [19]. This amendment is based on the study of the vehicular safety concepts, communication between vehicle and vehicle, and vehicle to road side unit. The performance of the 802.11 over the $802.11 \mathrm{p}$ will be discussed in this paper. This vehicular model with Manhattan mobility model and $802.11 \mathrm{p}$ standard is simulated using the NS2.34 version simulator [20]. The IEEE $802.11 \mathrm{p}$ technology can be used in NS2.33 and above versions only.

\subsection{VANET Routing protocols}

A routing protocol plays an essential role in vehicular network data communication. The routing protocols included in NS2.34 version are Destination Sequence Distance Vector (DSDV) protocol, Dynamic Source Routing (DSR) and Ad-hoc On-demand Distance Vector (AODV) routings protocol. The performance of the routing protocols in Manhattan mobility model is also discussed in this paper.

\subsection{Experimental analysis of Manhattan mobility model without RSUs}

In this paper the proposed concept is applied to the Manhattan mobility model and its performance is compared for standard 802.11 and $802.11 \mathrm{p}$. This experiment is repeated for various numbers of nodes. NAM file output for Manhattan mobility model without RSUs is shown in Figure 4.

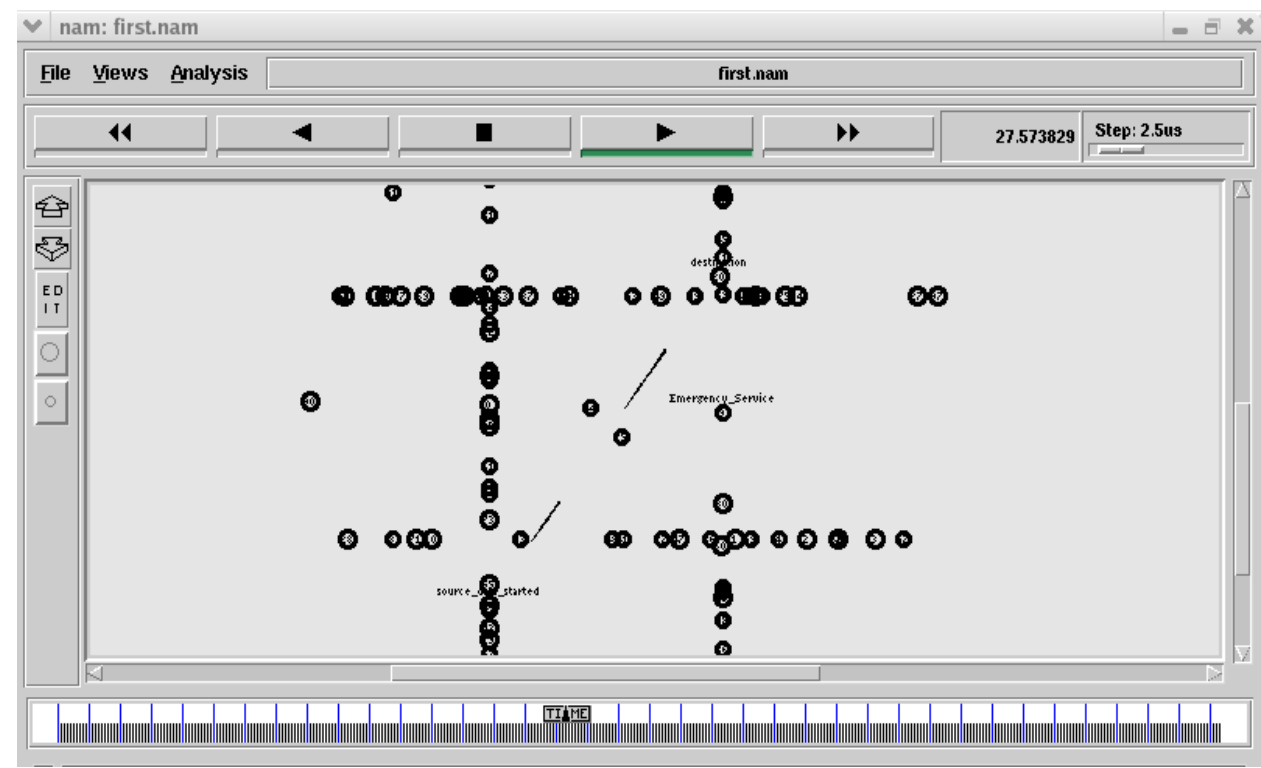

Figure 4: NAM file output for Manhattan model without RSUs

\subsection{Analysis of Packet receiving time for Manhattan mobility model}

From the Figure 5 it is noticed that better packet receiving time is achieved for DSDV routing protocol and 802.11p yields better performance than 802.11 using AODV protocol which is shown in Figure 6.

\subsection{Analysis of Routing over head and Normalized routing load for Manhattan model}

Figure 7 and 8 show that Routing over head is high for 25 nodes and this value decreases when the number of nodes increases. Normalized routing load is low for DSR and AODV protocol.

\subsection{Throughput for Manhattan model}

Figure 9 and 10 it is observed that the throughput for AODV protocol with 802.11 and $802.11 \mathrm{p}$ standard is higher than DSDV protocol. From Figure 11 it is clear that Throughput for $802.11 \mathrm{p}$ outperforms the 802.11 protocol. 


\subsection{Packet delivery ratio for Manhattan model}

From Figure 12, 13 and 14 it is noticed that for $802.11 \mathrm{p}$, AODV protocol yields better packet delivery ratio than DSDV protocol and Packet delivery ratio is high for 802.11p protocol and it is low for 802.11.

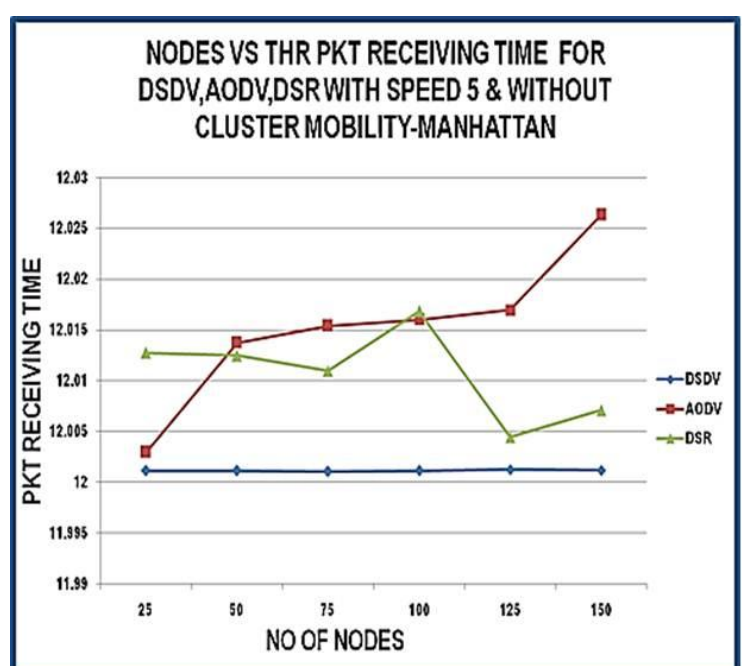

Figure 5: Packet receiving time for Manhattan model $\& 802.11 \mathrm{p}$

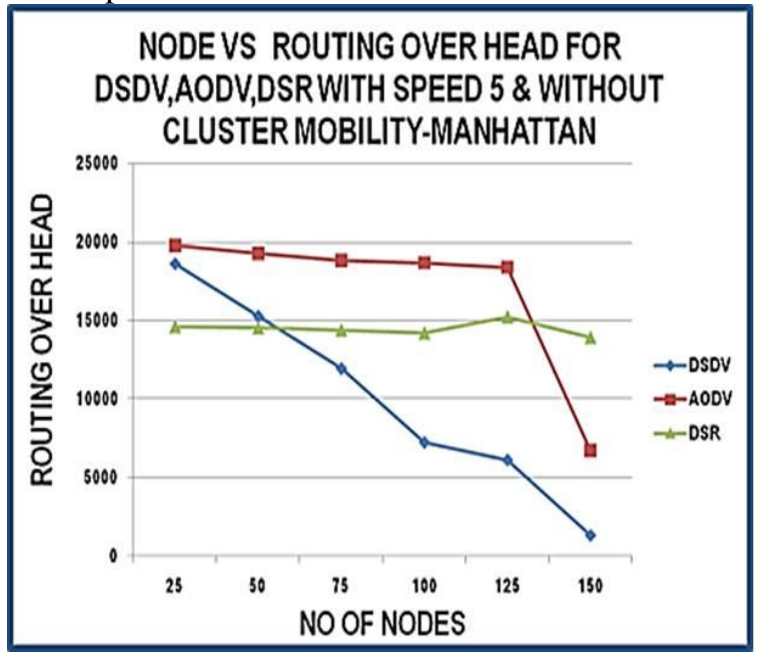

Figure 7: Packet overhead for Manhattan model

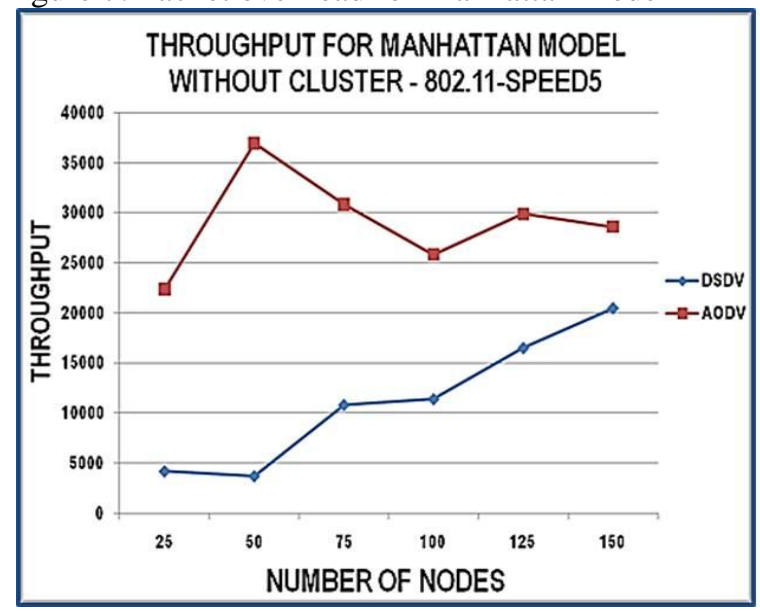

Figure 9: Packets Throughput using 802.11

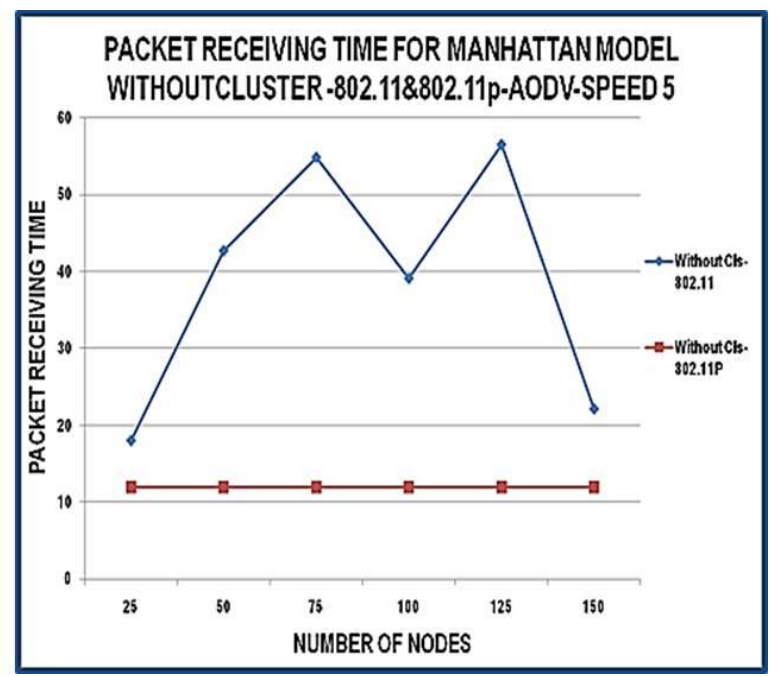

Figure 6: Packet receiving time using 802.11

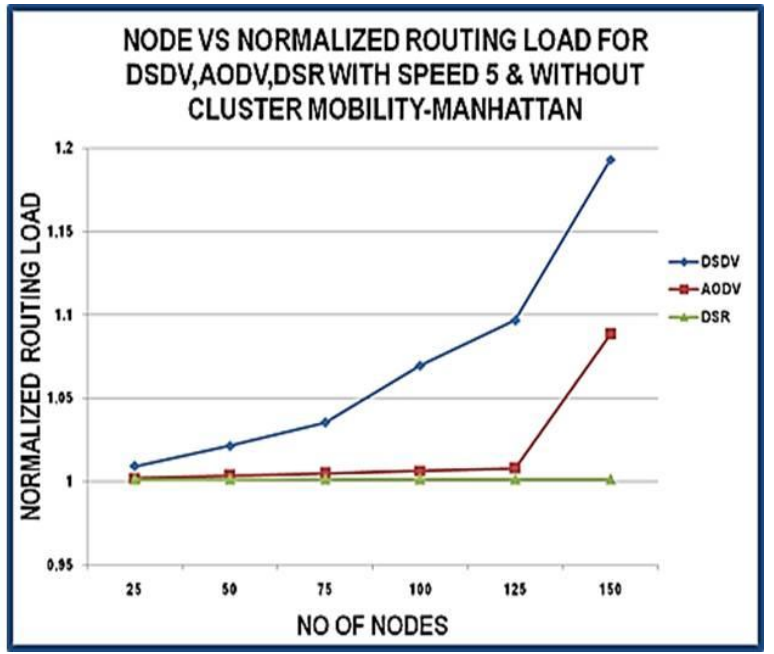

Figure 8: Normalized routing for Manhattan model

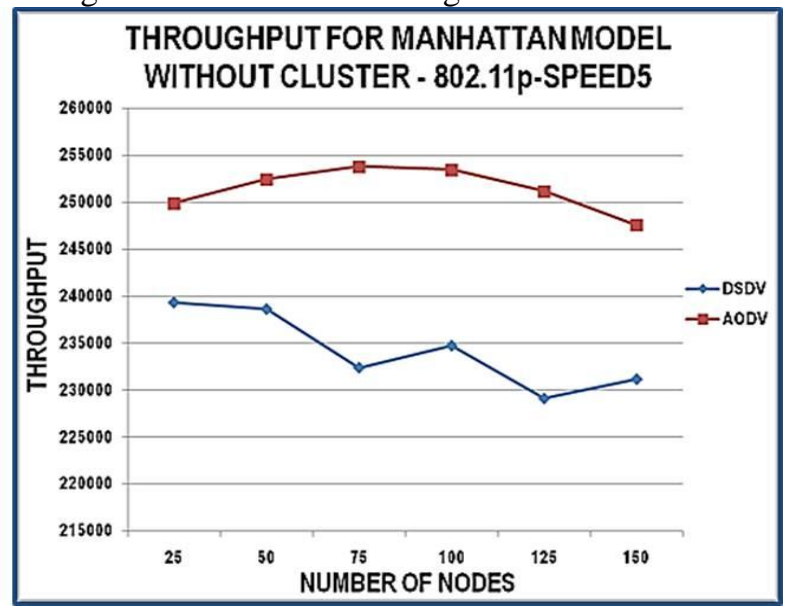

Figure 10: Packets Throughput using 802.11p 


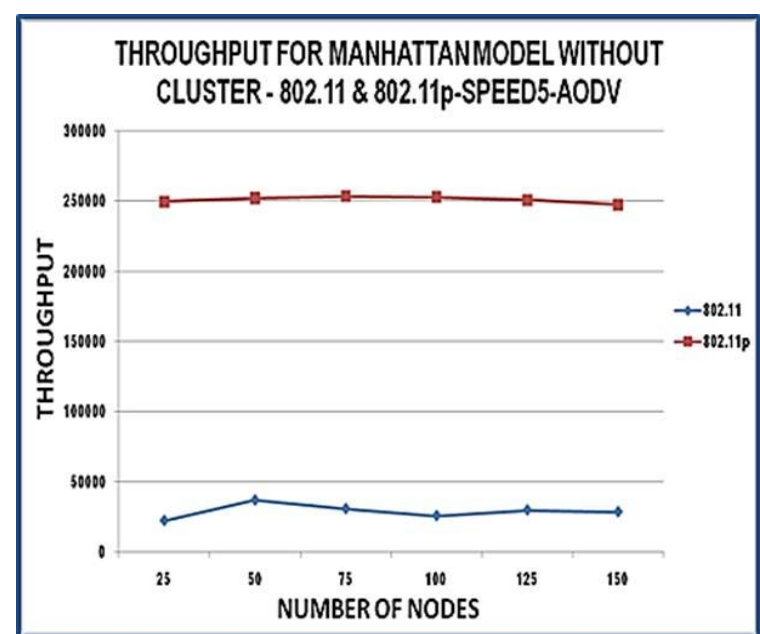

Figure 11: Throughput using 802.11p \&802.11

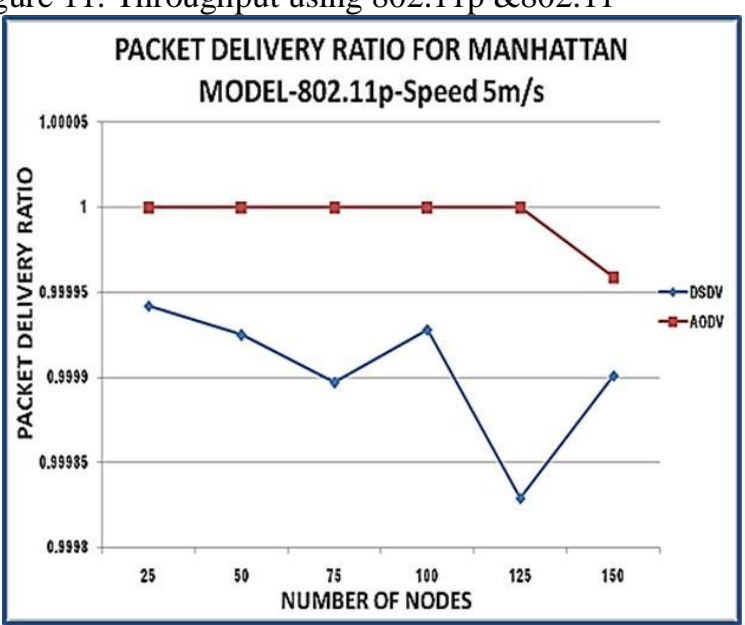

Figure 13: Packet Delivery ratio using $802.11 \mathrm{p}$ $802.11 \mathrm{p} / 802.11$

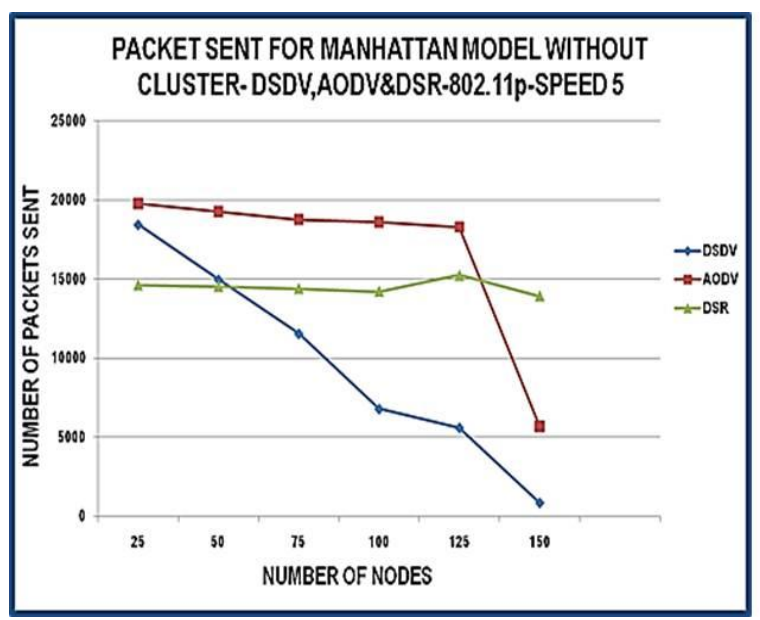

Figure 15: Packets sent for Manhattan model

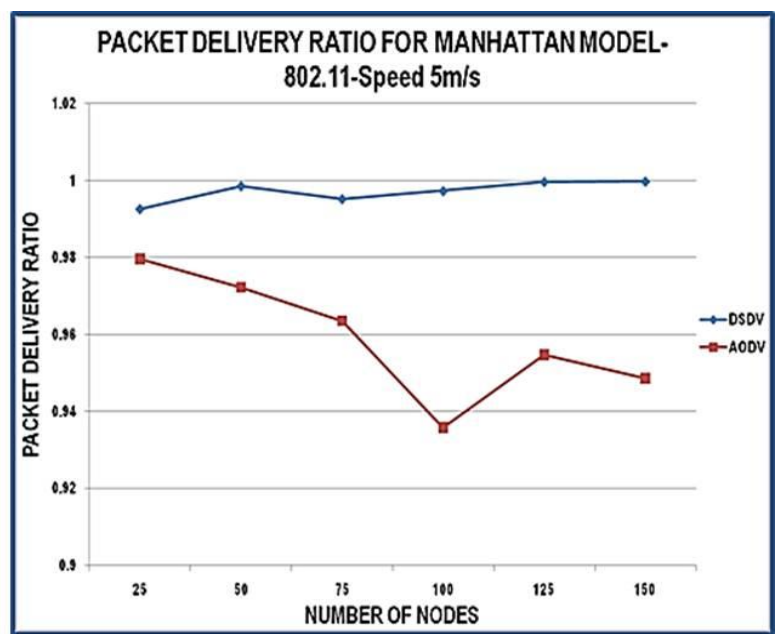

Figure 12: Packet Delivery ratio using 802.11

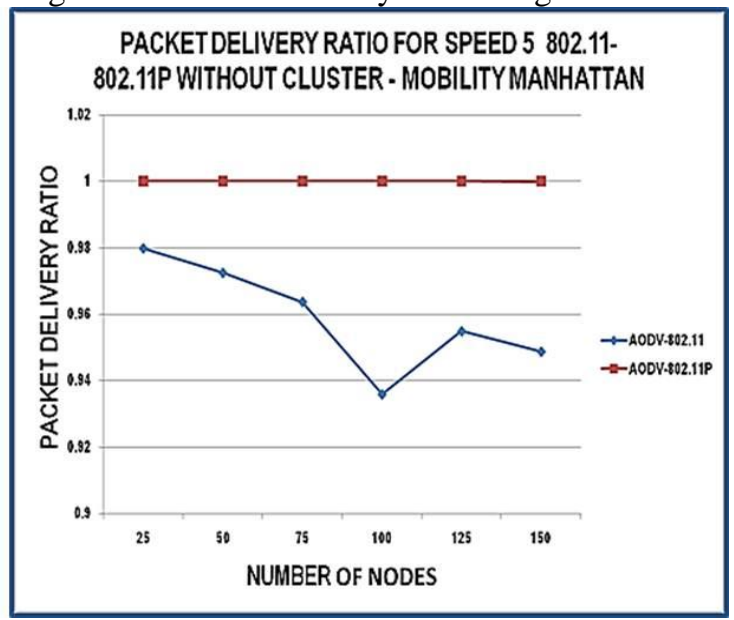

Figure14:Packet Delivery ratio using

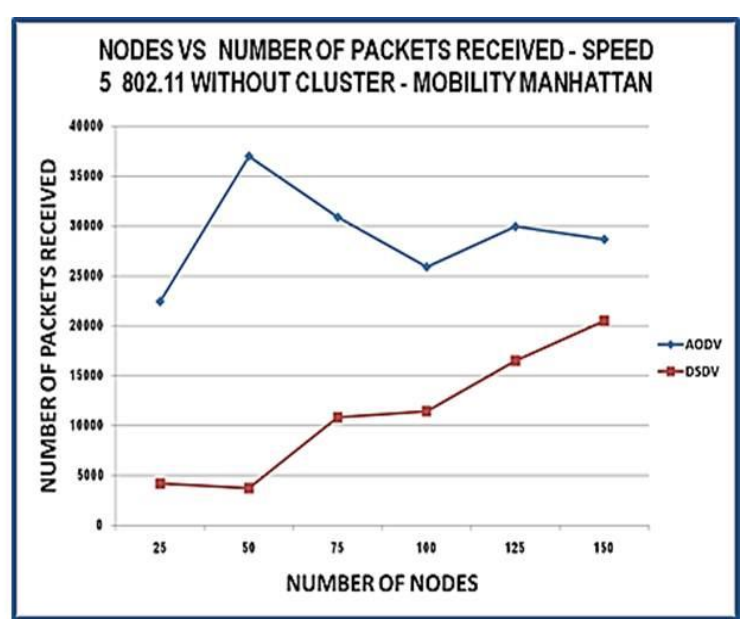

Figure 16: No of Packets received using 802.11 


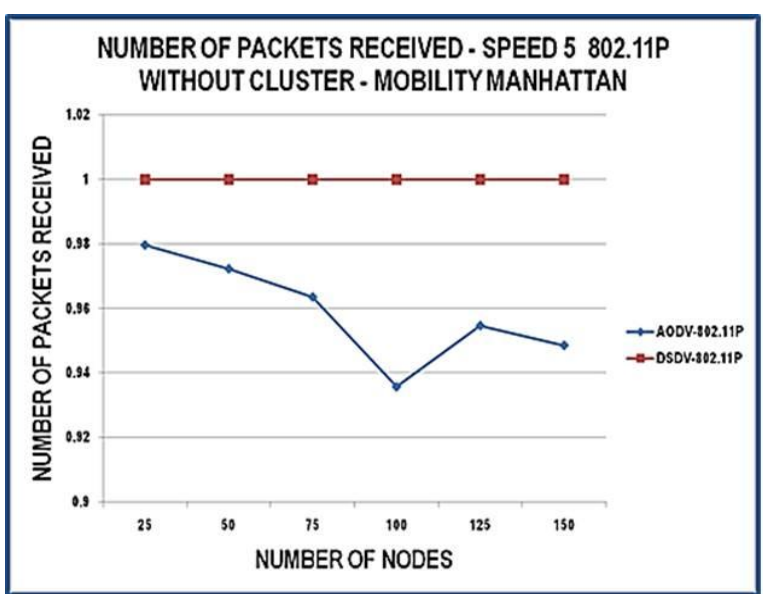

Figure 17: No of Packets received using 802.11p

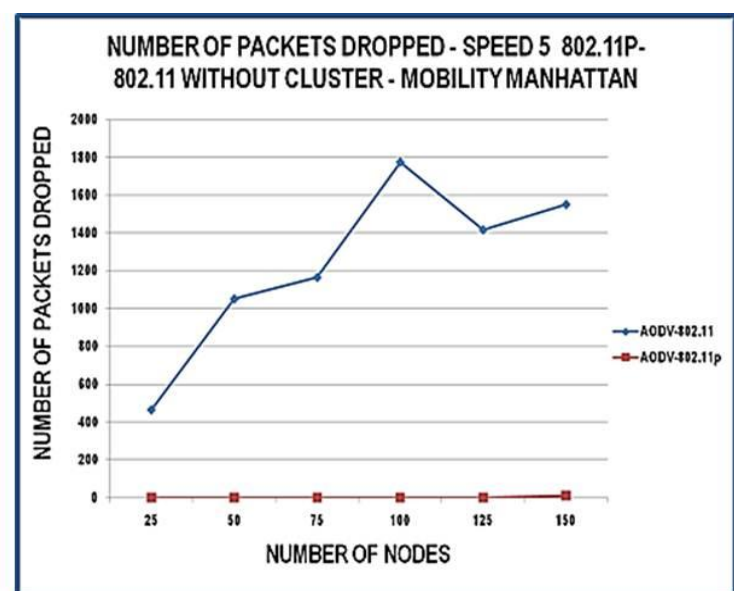

Figure18: No of Pkts Dropped using 802.11p/802.11

\subsection{Sent, Received and Dropped packets for Manhattan model}

The number of packets sent and received is high for AODV with $802.11 \mathrm{p}$ protocols. The number of packets dropped is low for $802.11 \mathrm{p}$ and it is high for 802.11 .

\begin{tabular}{|l|l|l|l|}
\hline Nodes & Packets sent & Packets received & Packets dropped \\
\hline 25 & 18448 & 18429 & 19 \\
\hline 50 & 14976 & 14956 & 20 \\
\hline 75 & 11555 & 11535 & 20 \\
\hline 100 & 6797 & 6777 & 20 \\
\hline 125 & 5688 & 5589 & 19 \\
\hline 150 & 863 & 843 & 20 \\
\hline
\end{tabular}

Table 2: Sent, Received and Dropped packets

\section{Conclusion}

Most of the earlier research works deal with the transaction based services provided by road side units. Moreover the earlier works concentrate on the Intelligent Transport System limited to the city areas where road side units are available in plenty. But this paper discusses the data communication without using the RSUs. The proposed concept is introduced in the Vehicular ad hoc network in which the movement of the vehicle is based on the Manhattan mobility model. The performance of routing protocols and standard 802.11 and $802.11 \mathrm{p}$ are discussed in this paper.

\section{References}

[1] Yong Hao, Yu Chengcheng, Chi Zhou, Wei Song, 'A Distributed Key Management Framework with Cooperative Message Authentication in VANETs', IEEE Journal on March 2011, Volume: 29, Page(s): 616-629.

Saeed, R.A. Naemat, A. Bin Aris, A. Bin Awang, M.K. Access Network Technol., Malaysia 'Design and evaluation of lightweight IEEE 802.11p-basedTDMA MAC method for road side -to-vehicle communications', The $12^{\text {th }}$ International Conference Advanced Communication Technology (ICACT), Feb. 2010 Volume: 2 , Page(s): 1483 - 1488.

[2] Routing in Vehicular Ad hoc Networks, 'A survey Vehicular Technology’ Magazine,IEEE Issue Date:June 2007 Volume: 2 Issue 2. Page(s): 12-22.

[3] Fan Bai Krishnan, 'Reliability Analysis of DSRC Wireless Communication for Vehicle Safety Applications', Intelligent Transportation Systems Conference, 2006. ITSC 06. IEEE Publication Year: 2006, Page(s): 355 - 362.

[4] Jerbi, M. Senouci, S.M. 'Characterizing Multi-Hop Communication in Vehicular Networks' Wireless Communications and Networking Conference, 2008. WCNC 2008. IEEE Publication Year: 2008, Page(s): 3309 - 3313.

[5] Fan Yu and Subir Biswas, 'Impacts of Radio Access Protocols on thePerformance of DSRC based ITS Applications', Proceeding of the $7^{\text {th }}$ International Conference on ITS Telecommunications, Jun. 2007. Page(s): 34-45

[6] T. Taleb, E. Sakhaee, K. Hashimoto, A. Jamalipour, N. Kato, and Y. Nemoto, 'A stable routing protocol to support ITS Services in VANET Networks,' IEEE Trans. on Vehicular Technology, Vol. 56, No. 6, Nov. 2007. Page: 3337-3347.

[7] Lin Yang Jingdong Xu Gongyi Wu Jinhua Guo, Nankai Univ Tianjin, China 'Road Probing: RSU Assisted Data Collection in Vehicular Networks' Wireless Communications, Networking and Mobile Computing, Beijing, Volume: 16, Issue 6. Page (s): 2 - 3.

[8] Saxena, N., Tsudik, G., Jeong Hyun Yi, Polytech. Univ., Brooklyn, 'Efficient Node Admission and Certificateless Secure Communication in Short-Lived MANETs', Parallel and Distributed Systems, IEEE Transactions - Feb. 2009, Volume: 20 Issue: 2 , Page(s): $158-170$.

[9] Morshed, M.M., Ko, F.I.S. Dongwook Lim, Rahman, M.H., Mazumder, M.R.R., Ghosh, J. 'Performance evaluation of DSDV and AODV routing protocols in Mobile Ad-hoc Networks', New Trends in Information Science and Service Science (NISS), 2010 4th International Conference on Publication Year: 2010 , Page(s): 399 - 403.

[10] Ali, S. Bilal, S.M. 'An Intelligent Routing protocol for VANETs in city environments', Computer, Control and Communication, 2nd International Conference on 2009, Page (s): $1-5$. 
[11] Zhao Ting Hao Jianjun Sun Li Li Jianfeng Ma Yan 'on mobility models in vehicularcommunication system' Broadband Network \& Multimedia Technology, 2009. IC-BNMT '09. 2nd IEEE International Conference on Oct-2009, Page(s): 57-60.

[12] Mobility_user-manual_pdf.

[13] Berijesh Kadri Mohandas, Amiya Nayak, Kshirasagar Naik, Nishith Goel 'ABSRP: A Service Discovery Approach for Vehicular Ad-Hoc Networks', Page(s): 1590-1594 IEEE Asia Pacific Service Computing Conference 2008.

[14] Shi, W., Liu Y 'Real-Time urban traffic monitoring with global positioning System equipped vehicles' Intelligent Transport System, IET, Issue Date: June 2010, Volume 4, Issue 2, Page (s): 113-120.

[15] Wern-Yarng, Shieh Wei-Hsun, Lee Shen ,'Analysis of the Optimum Configuration of Roadside Units and Onboard Units in Dedicated Short-Range Communication Systems', Intelligent Transportation Systems, IEEE Transactions on Issue Date : Dec. 2006 Volume: 7, Issue: 4, Page(s): $565-571$.

[16] David A. Maltz, 'On-Demand Routing in Multi-hop Wireless Mobile Ad Hoc Networks', Ph.D., dissertation.

[17] Qi Chen, Felix Schmidt Eisenlohr, DanielJiang, Marc Tollent-Moreno-Luca Delgrossi 'Overhaul of ieee 802.11 modeling and simulation in ns-2' ACM New York, NY, USA -2007, Page(s): $159-168$.

[18] Alasmary, Waleed Zhuang, Weihua 'The Mobility Impact in IEEE $802.11 \mathrm{p}$ Infrastructure less Vehicular Networks' Vehicular Technology Conference Fall (VTC 2010-Fall), 2010', Issue Date: 6-9 Sept.2010, Page(s) 1-5, Canada.

[19] Abrougui, K, 'Location-Aided Gateway Advertisement and Discovery Protocol for VANets' Vehicular Technology, IEEE Transactions onOct.2010, Volume: 59, Issue: 8, Page(s): 3843 - 3858.

\section{AUTHOR}

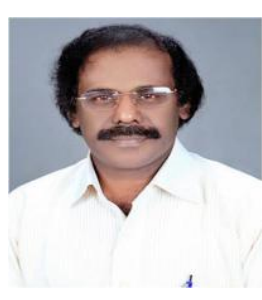

Dr. B. Ramakrishnan is currently working as Associate Professor in the Department of Computer Science and research centre in S.T. Hindu College, Nagercoil.

He received his M.Sc degree from Madurai Kamaraj university, Madurai and received Mphil (Comp. Sc.) from Alagappa University Karikudi. He earned his Doctorate degree in the field of Computer Science from Manonmaniam Sundaranar University, Tirunelveli. $\mathrm{He}$ has a teaching experience of 25 years. His research interests lie in the field of Vehicular networks, mobile network and communication, Cloud computing, Green computing and Ad-hoc networks. 